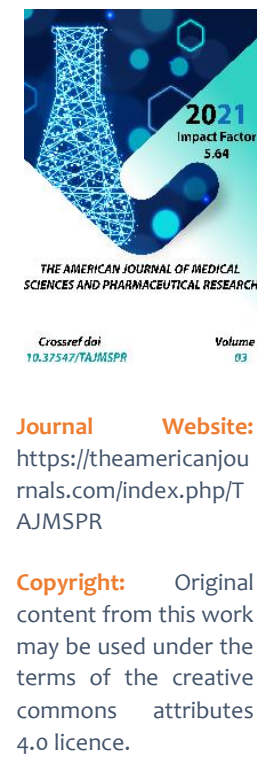

\section{The State Of The Cardiovascular System In Patients With Covid-19 In The Early Rehabilitation Period}

\author{
Daminova L.T. \\ Tashkent State Dental Institute, Uzbekistan \\ Daminov B. T. \\ Tashkent Pediatric Medical Institute, Uzbekistan \\ Muminov D.K. \\ Tashkent Pediatric Medical Institute, Uzbekistan \\ Davron Kadirovich Muminov \\ Department Off Faculty Internal Diseases, Occupatuonal Pathology, MFT, Hospital Internal \\ Diseases And PID, Tashkent Pediatric Medical Institute, Tashkent, Republic Of Uzbekistan
}

\title{
ABSTRACT
}

Purpose of the study: assessment of the study cardiovascular status in patients with postcovid syndrome and to determine their relationship with the functional status of patients.

Material and research methods. There were 281 patients under observation, with the presence of clinical manifestations of postcovid syndrome. The comparison group (CG) consisted of 20 patients who had undergone COVID-19 and whose disease ended in full recovery. All patients underwent electrocardiographic (ECG) and echocardiographic (EChoCG) studies. The assessment of the functional status of patients after suffering COVID-19 was carried out using the PCFS method - Post-COVID-19 Functional Status (https://osf.io/qgpdv/).

Research results. The study revealed signs of cardiovascular pathology according to ECG and EchoCG data in 255 (90.75\%) patients with postcovid syndrome. According to the ECG results, cardiac arrhythmias were recorded significantly more often in patients in the main group than in the comparison group ( $p<0.001$ ). The results of the echocardiography of the study showed that in the patients of the main group, compared with the comparison group, there was a significant increase in the size of the LV and RV of the heart ( $p<0.05)$, a decrease in LVEF $(p<0.01)$ and a higher value of the mean pressure in the PA $(p<0.05)$. Patients with a higher PCFS score are characterized by large sizes of both ventricles ( $p<0.05$ for LV and $p<0.01$ for RV) and low LV ejection fraction ( $p<0.001)$.

Conclusion. The present study showed that 255 (90.75\%) patients who underwent COVID-19 in the early rehabilitation period have signs of cardiovascular pathology according to ECG and EchoCG data. Patients with a higher PCFS score (significant restriction of daily activity due to symptoms associated with the previous infection and the need for assistance in self-care) had more enlarged sizes of both ventricles and a lower LVEF. 


\section{KEYWORDS}

COVID-19, cardiovascular status, PCFS scale.

\section{INTRODUCTION}

The pandemic of coronavirus infection COVID19 (CoronaVirus Disease-2019), caused by a new strain of coronavirus - SARS-CoV-2 (severe acute respiratory syndrome coronavirus-2), has caused a rapid increase in the number of cases and high mortality worldwide. Despite the tropism of SARS-CoV-2 to the lungs, with COVID-19 there is a high risk of developing multiple organ failure, including due to damage to the cardiovascular system (CVS) $[1,2]$.

The problem of cardiac comorbidity in COVID19 has several aspects: the effect of concomitant CVDs on the incidence of a new viral infection, the severity of its course and the risk of mortality, as well as possible side effects of a number of drugs traditionally prescribed for the treatment of certain types of CVD [3]. Patients who have undergone COVID-19, especially in moderate and severe forms, with CVS disorders, need medical rehabilitation. Formation of rehabilitation programs for such patients, taking into account their functional status [4].

\section{PURPOSE OF THE STUDY}

Assessment of the study cardiovascular status in patients with postcovid syndrome and to determine their relationship with the functional status of patients.

\section{MATERIAL AND RESEARCH METHODS}

There were 281 patients under observation, with the presence of clinical manifestations of postcoid syndrome. The inclusion criteria were: 1) virologically confirmed no earlier than 30 and later 7 days prior to inclusion in the COVID-19 study; 2) negative result of PCR studies for SARS-CoV-19 at the time of inclusion in the study; 3) age 16-75 years. As a comparison group (CG), 20 patients were examined who had undergone COVID-19 at the same time, in which the infection ended in full recovery. All patients underwent electrocardiographic (ECG) and echocardiographic (EchocG) studies. The ECG was performed on an automatic 6-channel electrocardiograph, in a horizontal position, in the morning, after 10 minutes of rest. EchoCG was performed on an ultrasound scanner equipped with a 5-7.5 MHz sector transducer. The study was carried out according to the standard technique, lying on the left side and on the back, according to the recommendations of the American Sonographic Association. The end diastolic diameters of the left and right ventricles (LV and RV ED) and the left atrial diameter (LA) were recorded, the mean pulmonary artery pressure (Pav PA) was calculated in relation to the duration of the expulsion flow acceleration time to the duration of the expulsion period on the pulmonary valve. Determination of LV systolic function was carried out by calculating the LV ejection fraction (LVEF) according to the standard formula (the percentage of the difference between the end diastolic and systolic volumes of the LV, calculated by the Teicholz formula, from the end diastolic volume of the LV. The assessment of the functional status of patients after suffering COVID-19 was carried out using the PCFS method - Post-COVID-19 Functional Status (https://osf.io/qgpdv/). This method uses a questionnaire completed by the patient 
himself or a form filled out by the doctor in the process of interviewing the patient. In the case of both options, a point assessment of the functional status of patients who underwent COVID-19 was determined [5].

\section{RESEARCH RESULTS AND DISCUSSION}

The study revealed in 255 patients (90.75\%) of the main group signs of cardiovascular pathology according to ECG and EchoCG data. According to the results of ECG examination, cardiac arrhythmias were recorded significantly more often in patients in the main group than in the comparison group (66.19\% and $25 \%$, chi square $13.3, \mathrm{p}<0.001)$. The identified rhythm disturbances were manifested in the form of sinus tachycardia in 163 patients, supraventricular paroxysmal tachycardia - 30 patients, paroxysmal atrial fibrillation - 5 patients, ventricular extrasystole
- 46 patients, ventricular extrasystole corresponded to IV-V class according to the Lown system - in 3 patients, mixed forms arrhythmias - in 22 patients. Conduction disturbances were observed in 123 (43.78\%) patients with postcoid syndrome and in $1(5 \%)$ patient from the comparison group. Conduction disturbances were noted in the form of atrioventricular and sinoatrial blockade of 1 and 2 degrees Mobits 1. Also, according to the results of ECG examination, signs of repolarization disorders were revealed in the main group of patients, with a 50-75\% probability of ischemia (in the form and magnitude of ST-T deviation). Outpatient ECG monitoring by the Holter method was required for 62 patients of the main group (22.06\%). All diagnosed ECG symptoms were significantly more often recorded in patients of the main group (Table 1).

The frequency of detection of various ECG syndromes of the cardiac component of the postcoid syndrome

\begin{tabular}{|l|l|l|l|}
\hline Pathology & Main group $(\mathrm{n}=281)$ & $\begin{array}{l}\text { Comparison group } \\
(\mathrm{n}=20)\end{array}$ & Chi square \\
\hline Arrhythmia & $186(66,19 \%)$ & $5(25 \%)$ & $13,31^{* * *}$ \\
\hline Blockades & $123(43,78 \%)$ & $1(5 \%)$ & $11,89^{* * *}$ \\
\hline Deviation ST-T & $84(29,89 \%)$ & $2(10 \%)$ & $4,05^{*}$ \\
\hline
\end{tabular}

Note: * - reliability of the chi square test: one sign - $\mathrm{p}<0.05$, two signs $-\mathrm{p}<0.01$, three signs - $\mathrm{p}$ $<0.001$.

The results of the echocardiography of the study found that in patients of the main group, who showed clinical signs of postcovid syndrome, compared with the comparison group, there was a significantly greater size of the LV and RV of the heart ( $p<0.05$ ), a lower $\operatorname{LVEF}(p<0.01)$ and higher value of pressure in the aircraft $(p<0.05)$. In general, in 81 patients of the main group (28.83\%), LVEF was below the reference norm of $55 \% .77$ patients (27.40\%) had moderate pulmonary hypertension. The echocardiography of the comparison group was comparable to that of healthy individuals (CG).

During the examination, in 69 patients (24.56\%) of the main group and in 2 patients (10\%) of the comparison group, a violation of regional contractility was found (the frequency difference is not significant). Also, 79 patients 
in the main group (28.11\%) and 2 patients in the comparison group (10\%, the difference between the groups was nd) had an effusion in the pericardial cavity, but in no case did the volume of effusion exceed $150 \mathrm{ml}$ (according to the Teicholz method) and did not noted clinic of cardiac tamponade. All patients included in the study underwent the PCFS test, which reflects the severity of the functional disorder of patients associated with a previous viral infection. In the main group of patients, the average score of the questionnaire was $13.34 \pm$ 0.83 points. There were 13 patients with postcovid syndrome who determined a violation of their functional status in 1 point (4.63\%), 2 points - 90 people (32.03\%), 3 points 117 people (41.64\%), 4 points - 61 people (21.71\%). The small number of patients who rated their condition disorders at 1 point is probably due to the method of recruiting patients - patients hospitalized due to poor health were included. 1 point according to the PCFS method indicates a slight limitation of daily activity, which does not motivate the patient to go to a rehabilitation hospital. In the comparison group, patients who underwent COVID-19 rated their functional status disorders due to infection at o points, which means that they have no restrictions on activity and complaints associated with the previous infection, including pain, anxiety, depression. In the course of the study, a comparison was made of patients in the main group, distributed depending on the severity of functional disorders. The comparison showed that patients with a higher PCFS score (significant limitation of daily activity due to symptoms associated with the previous infection and the need for assistance in self-care) have larger sizes of both ventricles ( $p<0.05$ significant difference for the LV and $p<0.01$ for RV) and low LVEF ( $p<0.001)$. EchoCG characteristics of patients with a PCFS score of 1-2 did not differ from those of the comparison group, except for a relatively reduced LV EF $(p<0.05)$. When conducting a correlation analysis, it was found that the value of LVEF in patients of the main group strongly negatively correlated with the PCFS score $(r=-0.73, p<0.01)$, which indicates a significant contribution of cardiovascular syndrome to a decrease in the functional status of patients who underwent COVID-19. According to the literature, the types of cardiovascular disorders vary widely: arrhythmias, myocardial damage and myocarditis, heart failure (HF) and cardiomyopathy, acute coronary syndrome (ACS) and myocardial infarction (MI), cardiogenic shock and cardiac arrest, venous thromboembolism [6, 7, 8]. Etiopathogenetic factors of cardiac arrhythmia and conduction disorders in COVID-19 can be hypoxia, hyperthermia, agitation, hypercatecholaminemia, electrolyte and metabolic disorders, myocardial damage, myocardial ischemia / infarction and, finally, side effects of drugs [8,9]. A viral infection can provoke decompensation of chronic heart failure (CHF). According to a Chinese study, the incidence of HF in deaths from COVID-19 was $52 \%$, and among survivors $-12 \%(p<0.0001)$, the incidence of new cases of HF in hospitalized patients with COVID-19 averaged 23\% [10]. Acute HF against a background of viral infection is obviously a consequence of previous systolic dysfunction, ARDS, and de novo cardiovascular pathology (acute myocardial ischemia, MI, tachyarrhythmias, myocarditis or cardiomyopathies) [11]. In a study by E. Argulian et al. found that dilatation of the right ventricle, detected by echocardiography (EchoCG), is associated with a high risk of hospital death in patients with COVID-19. Pulmonary hypertension developing 
in COVID-19 due to damage to the lungs and hypoxia or due to thromboembolism of the pulmonary arteries increases the load on the right ventricle, leading to damage to cardiomyocytes [12].

\section{CONCLUSION}

The present study showed that 255 (90.75\%) patients who underwent COVID-19 in the early rehabilitation period have signs of cardiovascular pathology according to ECG and EchoCG data. Patients with a higher PCFS score (significant limitation of daily activity due to symptoms associated with the previous infection and the need for assistance in selfcare) had more enlarged sizes of both ventricles and a lower $L V$ ejection fraction. This circumstance indicates a significant contribution of cardiovascular syndrome to a decrease in the functional status of patients who have undergone COVID-19.

\section{REFERENCES}

1. World Health Organization. Coronavirus disease 2019 (COVID-19) ituation report - 48. Available at: https://www.who.int/docs/default source/coronaviruse/situation-re ports/20200308-sitrep-48-covid-19 [Accessed: March 9, 2020].

2. Wang D., Hu B., Hu C. et al. Clinical characteristics of 138 hospitalized patients with 2019 novel coronavirusinfected pneumonia in Wuhan, China. JAMA. 2020; 323 (11): 1061-1069. DOI: 10.1001/jama.2020.1585.

3. Li B., Yang J., Zhao F. Prevalence and impact of cardiovascular metabolic diseases on COVID-19 in China. Clin Res Cardiol. 2020
4. Bansal M. Cardiovascular disease and COVID-19. Diabetes Metab Syndr. 2020;14(3):247-250. doi:10.1016/j.dsx.2020.03.013

5. Klok, F. A., Boon, G., Barco, S., Endres, M., Geelhoed, J., Knauss, S., Rezek, S. A., Spruit, M. A., Vehreschild, J., \& Siegerink, B. (2020). The Post-COVID-19 Functional Status scale: a tool to measure functional status over time after COVID-19. The European respiratory journal, 56(1), 2001494. https://doi.org/10.1183/13993003.01494 $-2020$

6. Aghagoli G., Gallo M. B., Soliman L. B. et al. Cardiac involvement in COVID-19 patients: Risk factors, predictors, and complications: A review // J. Card. Surg. -2020. - Apr 19. DOI: 10.1111/jocs.14538.

7. Chen C., Zhou Y., Wang D.W. SARS-CoV2: a potential novel etiology of fulminant myocarditis. Herz, 2020, vol. 45, no. 3, pp. 230-232. doi: 10.1007/s00059-020-04909-z.

8. Driggin E., Madhavan M.V., Bikdeli B. et al. Cardiovascular considerations for patients, health care workers, and health systems during the COVID-19 pandemic. J. Am. Coll. Cardiol., 2020, vol. 75 , no. 18 , pp. 2352-2371. doi: 10.1016/j.jacc.2020.03.031.

9. Kochi A.N., Tagliari A.P., Forleo G.B. et al. Cardiac and arrhythmic complications in patients with COVID19. J. Cardiovasc. Electrophysiol., 2020, vol. 31, no. 5, pp. 1003-1008. doi: 10.1111/jce.14479.

10. Zhou F., Yu T., Du R. et al. Clinical course and risk factors for mortality of adult in patients with COVID-19 in Wuhan, China: a retrospective cohort study. 
Lancet. 2020; 395 (10229): 1054-1062.

DOI: 10.1016/S0140-6736(20)30566-3.

11. Hendren N.S., Drazner M.H., Bozkurt B., Cooper L.T. Description and proposed management of the acute COVID-19 cardiovascular syndrome. Circulation. 2020; 141 (23): 1903-1914. DOI:

10.1161/CIRCULATIONAHA.120.047349

12. Argulian E., Sud K., Vogel B. et al. Right ventricular dilation in hospitalized patients with COVID-19 infection. JACC Cardiovasc. Imaging. [Preprint. Posted 2020, May 15]. DOI: 10.1016/j.jcmg.2020.05.010. 